\title{
Gene co-expression network and function modules in three types of glioma
}

\author{
GANG LI, WEIRAN PAN, XIAOXIAO YANG and JINMING MIAO \\ Department of Neurosurgery, Shengjing Hospital of China Medical University, \\ Shenyang, Liaoning 110004, P.R. China
}

Received October 17, 2013; Accepted April 25, 2014

DOI: $10.3892 / \mathrm{mmr} .2014 .3014$

\begin{abstract}
The aim of the present study was to identify the disease-associated genes and their functions involved in the development of three types of glioma (astrocytoma, glioblastoma and oligodendroglioma) with DNA microarray technology, and to analyze their differences and correlations. First, the gene expression profile GSE4290 was downloaded from the Gene Expression Omnibus database, then the probe-level data were pre-processed and the differentially expressed genes (DEGs) were identified with limma package in $\mathrm{R}$ language. Gene functions of the selected DEGs were further analyzed with the Database for Annotation, Visualization and Integrated Discovery. After the co-expression network of DEGs was constructed by Cytoscape, the functional modules were mined and enrichment analysis was performed, and then the similarities and differences between any two types of glioma were compared. A total of 1151 genes between normal and astrocytoma tissues, 684 genes between normal and malignant glioma tissues, and 551 genes between normal and oligodendroglioma tissues were filtered as DEGs, respectively. By constructing co-expression networks of DEGs, a total of 77232, 455 and 987 interactions were involved in the differentially co-expressed networks of astrocytoma, oligodendroglioma and glioblastoma, respectively. The functions of DEGs were consistent with the modules in astrocytoma, glioblastoma and oligodendroglioma, which were mainly enriched in neuron signal transmission, immune responses and synthesis of organic acids, respectively. Model functions of astrocytoma and glioblastoma were similar (mainly related with immune response), while the model functions of oligodendroglioma differed markedly from that of the other two types. The identification of the associations among these three
\end{abstract}

Correspondence to: Dr Weiran Pan, Department of Neurosurgery, Shengjing Hospital of China Medical University, 36 Sanhao Street, Heping, Shenyang, Liaoning 110004, P.R. China

E-mail: weiranpan@hotmail.com

Key words: glioma, gene expression profiling, differentially expressed gene, co-expression network, pathway enrichment analysis, function module mining types of glioma has potential clinical utility for improving the diagnosis of different types of glioma in the future. In addition, these results have marked significance in studying the underlying mechanisms, distinguishing between normal and cancer tissues, and examining novel therapeutic strategies for patients with glioma.

\section{Introduction}

Glioma derived from glial cells is the most common type of primary intracerebral neoplasm, accounting for $>40 \%$ of all primary brain tumors in humans (1), and typically has a poor prognosis (2). The classification of glioma varies depending on different standards. In the present study, the three most prevalent types of glioma that exhibit a high degree of malignancy were selected for in-depth analysis, astrocytoma, oligodendroglioma and glioblastoma. Astrocytoma has the highest morbidity; oligodendroglioma, which originates from the interstitial cells is more common than the neuronal tumor that originates from the parenchyma cells; while the medical history of glioblastoma is notably short (3).

Glioma has been the subject of an increasing number of studies over the past two decades, that has resulted in considerable advances in the understanding of basic biology and pathogenesis $(3,4,5)$. This accumulative evidence is contributing to development of novel pharmacological strategies to target the key molecular pathways involved in gliomas and reduce the resulting mortality and morbidity by 2020 (6). Despite aggressive treatment with surgery, radiation and chemotherapy, the average survival time for glioma patients remains only 14 months (7). Therefore, more effective therapies are urgently required. Furthermore, due to the progressive nature of cancer, which involves multiple mutations, individual gene analysis may only provide limited insights into the cellular mechanisms underlying carcinogenesis (8). Therefore, further investigation of the pathogenetic basis of glioma is imperative.

In recent years, with the continuous development of biotechnology and the innovation of novel high throughput technology, studies have begun to investigate the occurrence and development of diseases at the genome level, and gene chip technology is commonly used to detect genome-wide gene expression at the transcriptome level. Numerous studies have screened biomarkers associated with diseases by analyzing gene expression profiling $(9,10)$, and have analyzed the 
pathogenesis of various complex diseases (11) by associating the gene expression level with biological networks, including protein interaction networks (12), regulation and metabolic networks (13) and signal networks (14). The present study aimed to identify the disease-associated genes and gene functions in three types of glioma (astrocytoma, glioblastoma and oligodendroglioma) based on DNA microarray gene expression profiling and to analyze their differences and correlations, which may provide novel avenues for more advanced investigation into the mechanisms underlying glioma. Improving the understanding of the molecular mechanisms of glioma may provide evidence that aids in the development of early diagnosis and treatment strategies for patients.

\section{Materials and methods}

Microarray data. Gene Expression Omnibus (GEO) (15) (http://www.ncbi.nlm.nih.gov/geo/) is currently the largest and most comprehensive public gene expression database. The gene expression data stored in GEO is mainly submitted from laboratories worldwide, including Affymetrix microarray data, expressed sequence tag sequence data, serial analysis of gene expression sequence data and second generation sequence data. Each set of the submitted expression profile data include information of platforms, sequences and samples. The gene expression profile GSE4290 (16) was downloaded from the GEO database, including 157 glioma tissue samples: 26 Astrocytoma, 81 glioblastoma, 50 oligodendroglioma and 23 normal tissue samples.

Data preprocessing. According to the corresponding correlation between the probe and gene from the GPL570 platform [HG-U133_Plus_2](Affymetrix Human Genome U 133 plus 2.0 Array; Affymetrix, Santa Clara, CA, USA) the probe numbers of the expression profile were converted into the corresponding gene symbols. One gene corresponds with multiple probes, thus each gene has more than one expression value. Therefore, the average value was calculated and selected as the only representative value. A total of 20308 genes were involved following processing. Background correction and quartile data normalization were performed by the robust multiarray average in $\mathrm{R}$ affy package (Affymetrix).

Differentially expressed genes (DEGs) analysis. The limma package (Affymetrix)(17) was used to identify the DEGs between the normal and three glioma tissues, respectively. The cut-off criterion was $\mathrm{P}<0.01$.

Gene ontology (GO) enrichment analysis. For preliminary investigation into the functional differences of DEGs in the three types of glioma, Database for Annotation, Visualization and Integrated Discovery (DAVID) online software (DAVID Bioinformatics Resources 6.7 http://david.abcc.ncifcrf.gov/ home.jsp) was used to perform GO (18) enrichment analysis. DAVID (19) utilizes Fisher's exact test to enrich functions of certain genes. The cut-off criterion was the false discovery false corrected $\mathrm{P}<0.01$.

Co-expression network construction of DEGs. Multiple high-throughput expression data provide an opportunity for global research on gene functions. Genes involved in the same pathway or same functional compound, in different time and physiological conditions, tend to demonstrate a similar expression pattern (20). Therefore, the construction of a gene co-expression network facilitates the identification of gene collections with similar functions (21). Gene co-expression networks were constructed in the following procedure. Firstly, the expression values of normal samples were removed from the expression profile data. Secondly, Pearson's correlation coefficient between two DEGs was calculated according to the formula below, and the pairs whose correlation was $>0.7$ were selected as the co-expressed gene pairs:

$$
\mathrm{r}=\frac{1}{\mathrm{n}-1} \sum_{i=1}^{\mathrm{n}}\left(\frac{\mathrm{X}_{1}-\overline{\mathrm{X}}}{5_{\mathrm{X}}}\right)\left(\frac{\mathrm{Y}_{1}-\overline{\mathrm{Y}}}{\delta_{\mathrm{Y}}}\right)
$$

Where $\mathrm{r}$ is the Pearson's correlation coefficient; $\mathrm{n}$ is the sample size; $\bar{X}$ is the average of sample $\mathrm{X} ; \bar{Y}$ is the average of sample $\mathrm{Y} ; \delta_{X}$ is the standard deviation of sample $\mathrm{X}$; and $\delta_{Y}$ is the standard deviation of sample Y. Finally, clusterviz package in Cytoscape (22) was used to construct the co-expression network (1).

Function module mining and analysis. MCODE (23) was used to mine the function modules in the co-expression networks. The MCODE algorithm includes three steps: (i) The weight calculation of network vertex; (ii) the compound prediction; and (iii) the subsequent node filter or addition of the compound based on certain connection indicators. The network clustering coefficient $\mathrm{Ci}$ was selected as the node weight $\left[\mathrm{Ci}=2^{*} \mathrm{n} / \mathrm{Ki}^{*}(\mathrm{Ki}-1)\right.$, where $\mathrm{Ki}$ represents the node number directly connected with the node $\mathrm{i}$, and $\mathrm{n}$ represents the connected edge number of these nodes. The node with the largest weight was taken as seed to search the node $\mathrm{j}$ whose weight ratio $(\mathrm{Wj} / \mathrm{Wseed})$ was greater than a certain threshold. The value of the $\mathrm{j}$ node was considered, to repeat the search. A module was constituted until the threshold value was met. The searched nodes were removed from the network, so the searching number of each node was not $>1$. Next, the modules, which included $>10$ genes in the networks, were selected to perform the Gene Ontology enrichment analysis by DAVID.

\section{Results}

DEG analysis. A total of 1151 genes between normal and astrocytoma tissues, 684 genes between normal and glioblastoma tissues, and 551 genes between normal and oligodendroglioma tissues were filtered as DEGs, respectively.

Function enrichment of DEGs. Following the GO enrichment analysis, it was identified that the functions of DEGs in the three types of glioma were significantly different. The DEGs in astrocytoma were mainly involved in neuron signal transmission processes, including the synapse, synapse part and synaptic transmission (Table I). The DEGs of the glioblastoma were mainly involved in the immune response and antigen processing (Table II), and for oligodendroglioma, the DEGs were mainly involved in signal transmission processes, including GTPase activity and GTP binding (Table III). 
Table I. Enriched functions of DEGs in astrocytoma (top 10).

\begin{tabular}{llr}
\hline Category & \multicolumn{1}{c}{ Term } & FDR \\
\hline GOTERM_CC_FAT & GO:0045202 synapse & $2.04 \mathrm{E}-32$ \\
GOTERM_CC_FAT & GO:0044456 synapse part & $3.31 \mathrm{E}-30$ \\
GOTERM_BP_FAT & GO:0007268 synaptic transmission & $3.95 \mathrm{E}-24$ \\
GOTERM_BP_FAT & GO:0019226 transmission of nerve impulse & $1.35 \mathrm{E}-23$ \\
GOTERM_CC_FAT & GO:0008021 synaptic vesicle & $5.25 \mathrm{E}-19$ \\
GOTERM_CC_FAT & GO:0043005 neuron projection & $5.03 \mathrm{E}-18$ \\
GOTERM_BP_FAT & GO:0007267 cell-cell signaling & $5.27 \mathrm{E}-17$ \\
GOTERM_CC_FAT & GO:0044459 plasma membrane part & $3.11 \mathrm{E}-15$ \\
GOTERM_CC_FAT & GO:0005886 plasma membrane & $6.22 \mathrm{E}-13$
\end{tabular}

FDR, false discovery rate; DEGs, differentially expressed genes; GO, Gene ontology.

Table II. Enriched functions of DEGs in glioblastoma (top 10).

\begin{tabular}{llr}
\hline Category & \multicolumn{1}{c}{ Term } & \multicolumn{1}{c}{ FDR } \\
\hline GOTERM_BP_FAT & GO:0006955 immune response & $5.61 E-09$ \\
GOTERM_BP_FAT & GO:0048002 antigen processing and presentation of peptide antigen & $1.64 \mathrm{E}-06$ \\
GOTERM_BP_FAT & GO:0019882 antigen processing and presentation & $4.60 \mathrm{E}-06$ \\
KEGG_PATHWAY & hsa05416:Viral myocarditis & $3.44 \mathrm{E}-04$ \\
GOTERM_CC_FAT & GO:0042611 MHC protein complex \\
KEGG_PATHWAY & hsa04612:Antigen processing and presentation & $4.49 \mathrm{E}-04$ \\
GOTERM_CC_FAT & GO:0000323 lytic vacuole & $2.53 \mathrm{E}-03$ \\
GOTERM_CC_FAT & GO:0005764 lysosome & $3.52 \mathrm{E}-03$ \\
GOTERM_CC_FAT & GO:0042612 MHC class I protein complex \\
KEGG_PATHWAY & hsa05330:Allograft rejection & $3.52 \mathrm{E}-03$ \\
\hline
\end{tabular}

FDR, false discovery rate; DEGs, differentially expressed genes; GO, Gene ontology.

Table III. Enriched functions of DEGs in oligodendroglioma (top 10).

\begin{tabular}{lll}
\hline Category & \multicolumn{1}{c}{ Term } & \multicolumn{1}{c}{ FDR } \\
\hline GOTERM_MF_FAT & GO:0003924 GTPase activity & 1.282379 \\
GOTERM_MF_FAT & GO:0005525 GTP binding & 3.882750 \\
GOTERM_CC_FAT & GO:0043233 organelle lumen & 3.790407 \\
GOTERM_CC_FAT & GO:0031974 membrane-enclosed lumen & 3.855442 \\
GOTERM_BP_FAT & GO:0032989 cellular component morphogenesis & 5.467166 \\
GOTERM_MF_FAT & GO:0032561 guanyl ribonucleotide binding & 5.117207 \\
GOTERM_MF_FAT & GO:0019001 guanyl nucleotide binding & 5.117207 \\
GOTERM_BP_FAT & GO:0000902 cell morphogenesis \\
GOTERM_MF_FAT & GO:0008134 transcription factor binding & 8.180084 \\
GOTERM_CC_FAT & GO:0070013 intracellular organelle lumen & 7.159959 \\
\end{tabular}

FDR, false discovery rate; DEGs, differentially expressed genes; GO, Gene ontology.

Co-expression networks of DEGs. Co-expression networks of the three types of glioma were constructed, and the genes with a Pearson's correlation coefficient $>0.7$ were selected as the co-expressed gene pairs. A total of 77232, 455 and 987 interactions were involved in the differentially co-expressed networks of astrocytoma (Fig. 1), oligodendroglioma (Fig. 2) and glioblastoma (Fig. 3), respectively. From the three co-expression networks it was observed that they all had 
Table IV. Enriched functions of astrocytoma modules.

\begin{tabular}{|c|c|c|c|}
\hline Module & Function ID & Description & P-value \\
\hline \multirow[t]{15}{*}{ Module1 } & GO:0022836 & Gated channel activity (MF) & $3.549 \mathrm{E}-13$ \\
\hline & GO:0022839 & Iron gated channel activity (MF) & $3.549 \mathrm{E}-13$ \\
\hline & GO:0005216 & Iron channel activity (MF) & $5.003 \mathrm{E}-12$ \\
\hline & GO:0022838 & Substrate-specific channel activity (MF) & $9.450 \mathrm{E}-12$ \\
\hline & GO:0005215 & Transporter activity (MF) & $1.099 \mathrm{E}-11$ \\
\hline & GO:0007268 & Synaptic transmission (BP) & $3.940 \mathrm{E}-46$ \\
\hline & GO:0035637 & Multicellular organismal signaling (BP) & $1.496 \mathrm{E}-44$ \\
\hline & GO:0019226 & Transmission of nerve impulse (BP) & $3.705 \mathrm{E}-43$ \\
\hline & GO:0007267 & Cell-cell signaling (BP) & $3.354 \mathrm{E}-39$ \\
\hline & GO:0050877 & Neurological system process (BP) & $5.294 \mathrm{E}-35$ \\
\hline & GO:0045202 & Synapse (CC) & $1.230 \mathrm{E}-41$ \\
\hline & GO:0097458 & Neuron part (CC) & $1.414 \mathrm{E}-37$ \\
\hline & GO:0044456 & Synapse part (CC) & $6.823 \mathrm{E}-35$ \\
\hline & GO:0043005 & Neuron projection (CC) & $1.610 \mathrm{E}-33$ \\
\hline & GO:0008021 & Synaptic vesicle (CC) & $4.156 \mathrm{E}-22$ \\
\hline \multirow[t]{12}{*}{ Module2 } & GO:0070891 & Lipoteichoic acid binding (MF) & $3.231 \mathrm{E}-3$ \\
\hline & GO:0019864 & IgG binding (MF) & $4.791 \mathrm{E}-2$ \\
\hline & GO:0006955 & Immune response (BP) & $1.753 \mathrm{E}-13$ \\
\hline & GO:0006952 & Defense response (BP) & $5.365 \mathrm{E}-12$ \\
\hline & GO:0002682 & Regulation of immune system process (BP) & $1.062 \mathrm{E}-10$ \\
\hline & GO:0050776 & Regulation of immune response (BP) & $1.538 \mathrm{E}-10$ \\
\hline & GO:0045087 & Innate immune response (BP) & $2.973 \mathrm{E}-8$ \\
\hline & GO:0044420 & Extracellular matrix part (CC) & $2.914 \mathrm{E}-7$ \\
\hline & GO:0005764 & Lysosome (CC) & 4.999E-6 \\
\hline & GO:0000323 & Lytic vacuole (CC) & $4.999 \mathrm{E}-6$ \\
\hline & GO:0042611 & MHC protein complex (CC) & $9.777 \mathrm{E}-6$ \\
\hline & GO:0005773 & Vacuole (CC) & $2.442 \mathrm{E}-5$ \\
\hline
\end{tabular}

MF, molecular function; BP, biological process; CC, cellular component; GO, Gene ontology.

significantly different modularity, so their functions exhibited different emphases.

Function module mining and analysis. The MCODE method was used to mine the network modules. A total of 32, 7 and 10 modules were excavated from the differential co-expression networks of astrocytoma, glioblastoma and oligodendroglioma, respectively. The five modules with the maximum number of genes selected from the networks of the three types of glioma are demonstrated in Fig. 4. The modules that included $>10$ genes in the networks of the three types of glioma were selected for GO enrichment analysis.

The functions of the five modules of astrocytoma showed the diverse regulation of astrocytoma: Modulel was mainly involved in the function of neural signal transmission, including synaptic transmission, multicellular organismal signaling and transmission of nerve impulse. The functions of Module2 were mainly associated with the immune response, defense response and regulation of immune system processes. The functions of Module3 were mainly correlated with the myelin sheath. The functions of Module4 were mainly associated with the cell cycle and Module5 was mainly involved in signal transmission functions. Among them, the functions of Module1 and Module2 were summarized in Table IV. PAK3 p21-activated kinase 3 (PAK3) and docking protein 6 (DOK-6) were two genes in these modules.

Only two modules of the glioblastoma network contained $>10$ genes, and their enriched functions are demonstrated in Table V. Module1 was mainly enriched in functions related to immune response, regulation of immune responses and defense responses. Module2 was mainly enriched in biological processes, including cytoskeletal anchoring at plasma membrane, protein phosphorylation and regulation of sodium ion transport. CD86 and WNK lysine deficient protein kinase 2 (WNK2) were two genes in these modules.

Only one module was excavated with $>10$ genes in the differentially co-expressed network of oligodendroglioma, and it was identified that its functions were mainly enriched in the synthesis of organic acids. Cyclin-dependent kinase 5 (Cdk5) and DOK-6 were two genes in the module.

\section{Discussion}

Glioma is a multi-stage process, involving a variety of changes in gene expression and physiological structure, such as the 
Table V. Enriched functions of glioblastoma modules.

\begin{tabular}{lllc}
\hline Module & Function ID & \multicolumn{1}{c}{ Description } & P-value \\
\hline Module1 & GO:0019864 & IgG binding (MF) & $2.942 \mathrm{E}-2$ \\
& GO:0032395 & MHC class II receptor activity (MF) & $4.304 \mathrm{E}-2$ \\
& GO:0006955 & Immune response (BP) & $8.795 \mathrm{E}-10$ \\
& GO:0050776 & Regulation of immune response (BP) & $7.431 \mathrm{E}-8$ \\
& GO:0006952 & Defense response (BP) & $2.940 \mathrm{E}-7$ \\
& GO:0045087 & Innate immune response (BP) & $1.039 \mathrm{E}-6$ \\
& GO:0002253 & Activation of immune response (BP) & $1.754 \mathrm{E}-5$ \\
& GO:0005887 & Integral to plasma membrane (CC) & $8.919 \mathrm{E}-5$ \\
& GO:0031226 & Intrinsic to plasma membrane (CC) & $1.333 \mathrm{E}-4$ \\
& GO:0005764 & Lysosome (CC) & $9.638 \mathrm{E}-3$ \\
& GO:0000323 & Lytic vacuole (CC) & $9.638 \mathrm{E}-3$ \\
& GO:0044437 & Vacuolar part (CC) & $2.198 \mathrm{E}-2$ \\
& GO:0070853 & Myosin VI binding (MF) & $2.404 \mathrm{E}-2$ \\
Module2 & GO:0005200 & Structural constituent of cytoskeleton (MF) & $3.074 \mathrm{E}-2$ \\
& GO:0007016 & Cytoskeletal anchoring at plasma membrane (BP) & $2.365 \mathrm{E}-3$ \\
& GO:0046777 & Protein autophosphorylation (BP) & $1.682 \mathrm{E}-2$ \\
& GO:0002028 & Regulation of sodium ion transport (BP) & $4.280 \mathrm{E}-2$ \\
\hline
\end{tabular}

MF, molecular function; BP, biological process; CC, cellular component; GO, Gene ontology.

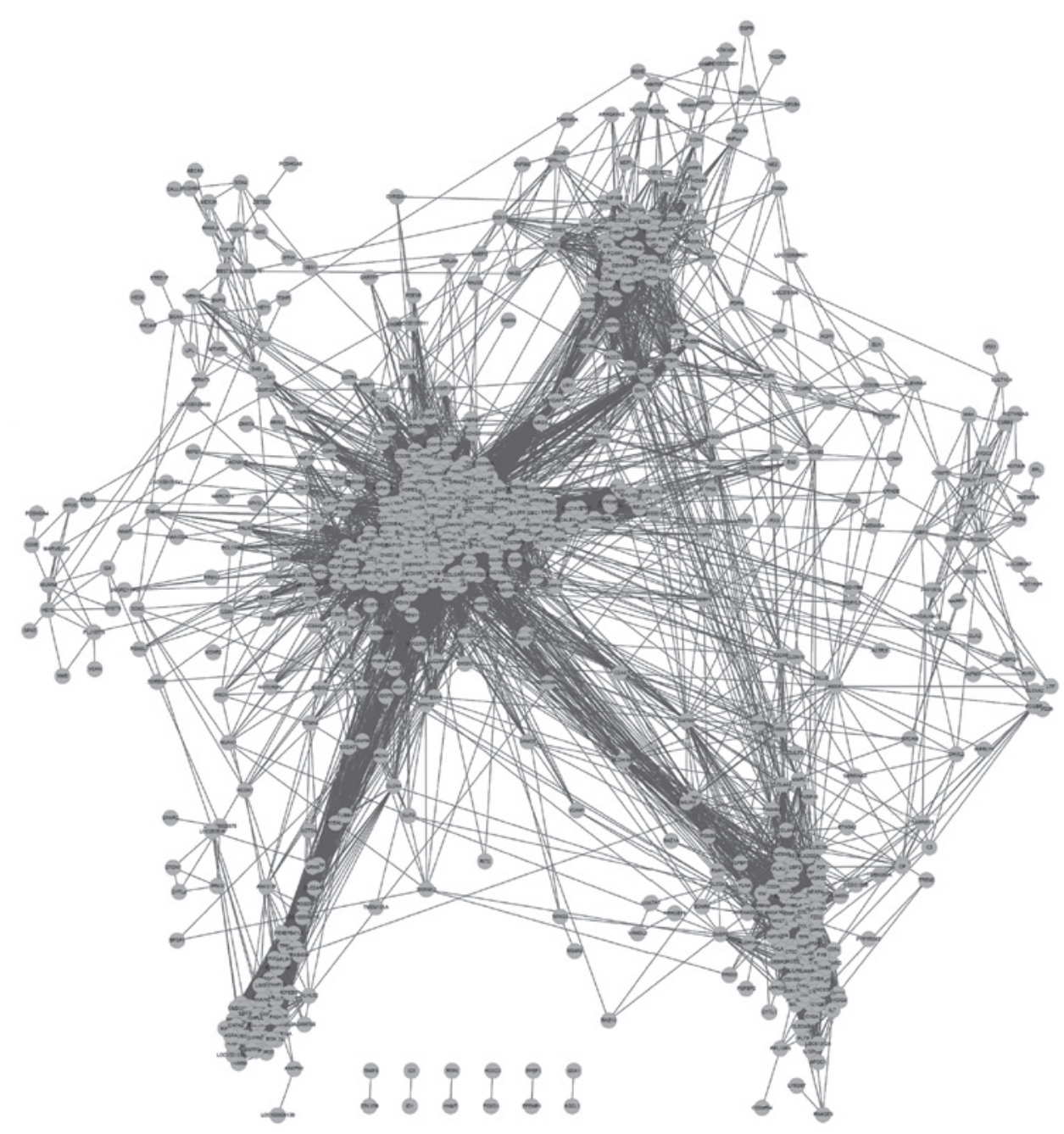

Figure 1. Differentially co-expressed network of differentially expressed genes in astrocytoma. 


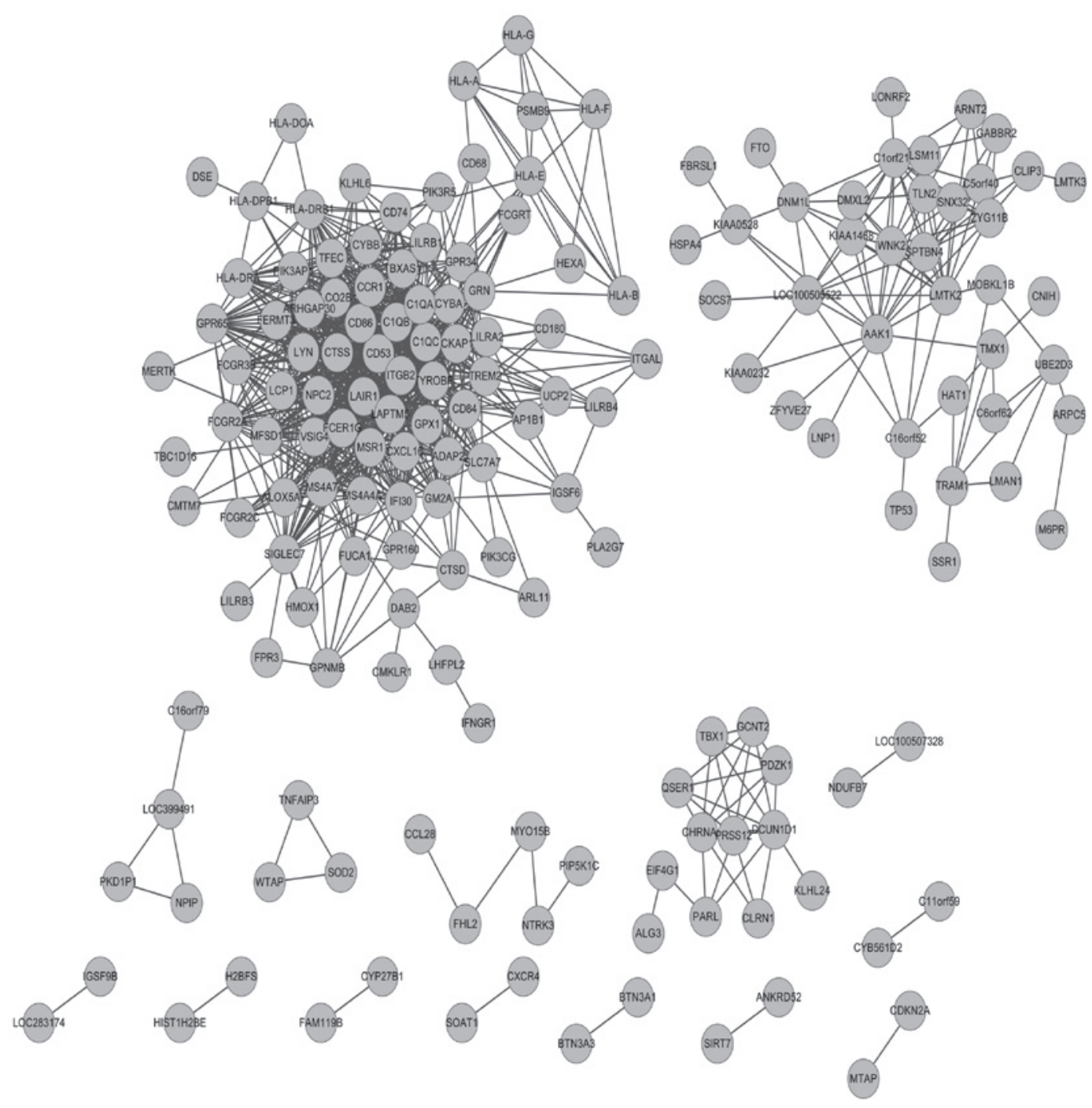

Figure 2. Differentially co-expressed network of differentially expressed genes glioblastoma.

expansion of suppressor cells, and is associated with high rates of patient mortality and morbidity (24). Therefore, examining the mechanism of glioma development and developing an effective prevention strategy is urgently required. In the present study, based on the gene expression profiling, DEGs associated with the three types of glioma (astrocytoma, glioblastoma and oligodendroglioma) were screened and co-expression networks were constructed. Next, the function modules were mined to determine the functional connections and differences in the DEGs in the three types of glioma. Based on the enrichment analysis of DEGs and modules, it was identified that the functions of DEGs were consistent with the modules in the three types of glioma. Therefore, DEGs in astrocytoma were mainly correlated with synaptic signaling, while those in glioblastoma were most commonly associated with protein phosphorylation and sodium ion transportation. The DEGs in the tissues from the two types of glioma had marked similarity, which was manifested by their relevance to immune response function. Conversely, DEGs in oligodendroglioma tissues were observed to be significantly difference compared with the other two types of glioma, as they were mainly associated with synthesis of organic acids; therefore, different treatment strategies may be required to treat this type of glioma.
Tumors arise through a combination of tumor-promoting inflammation and a failure of spontaneous antitumor immunity. Tumors have also been shown to initiate due to alterations in immune response genes in the absence of germline mutations in known oncogenes or anti-oncogenes. In addition, antitumor immunity has been shown to limit tumor growth (25). However, chronic immune activation also leads to the induction of immune-regulatory responses that promote tumorigenesis (25). Cancers are highly difficult for the immune system to eliminate as it is difficult to distinguish between healthy and tumor tissues. Those that have already escaped natural immunosurveillance have bypassed the natural immune barriers to malignancy (26). It may be concluded from the present study that the DEGs in astrocytoma and glioblastoma tissues were associated with the immune response. Therefore, understanding the molecular mechanisms (27) that separate tumor-promoting inflammation from antitumor immunity in tumor development may provide insights into inflammation-associated malignancies and contribute to the generation of immune therapies for cancer.

PAK3, which was involved in the co-expression network of the DEGs identified in astrocytoma tissues, has been 


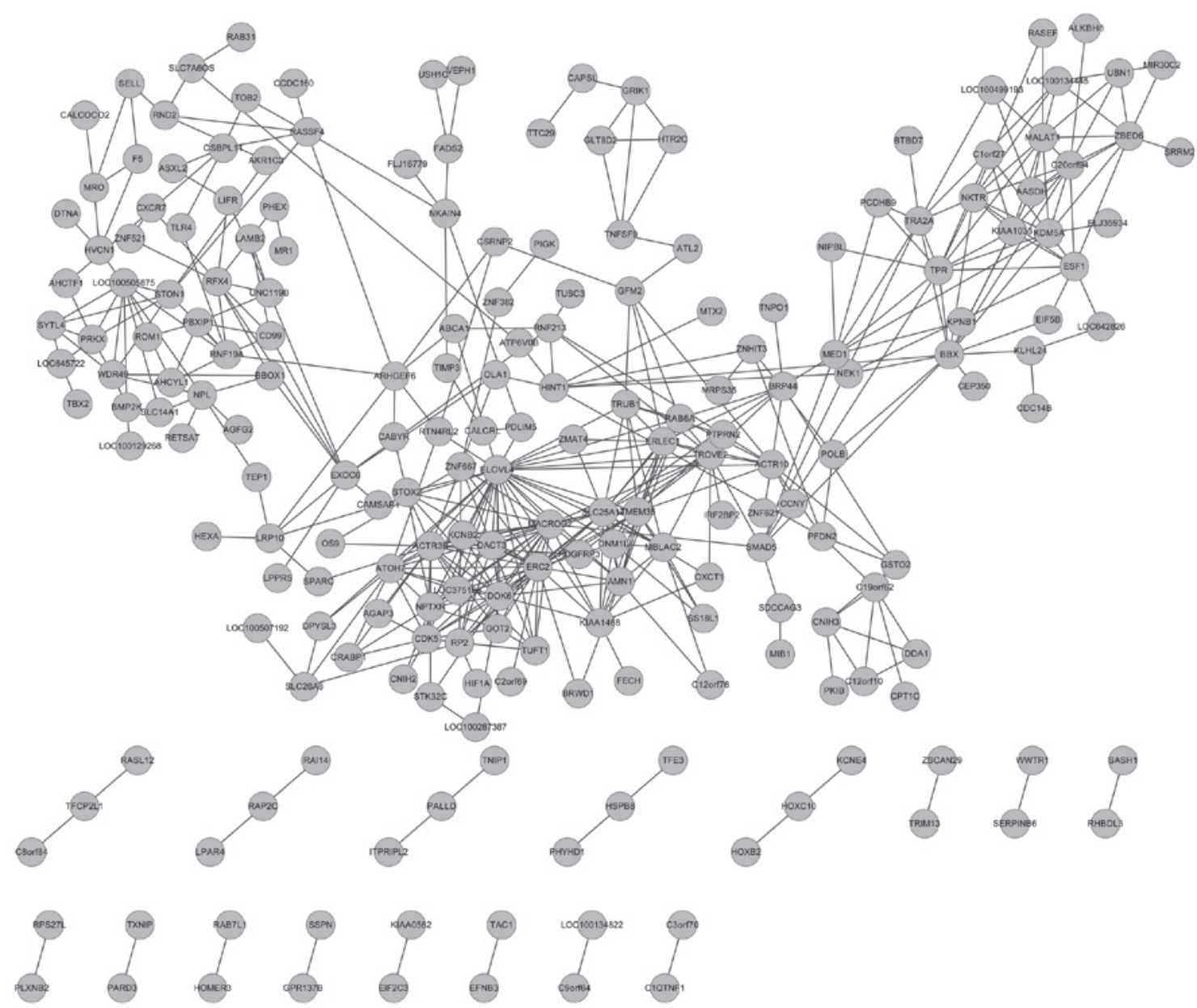

Figure 3. Differentially co-expressed network of differentially expressed genes oligodendroglioma.
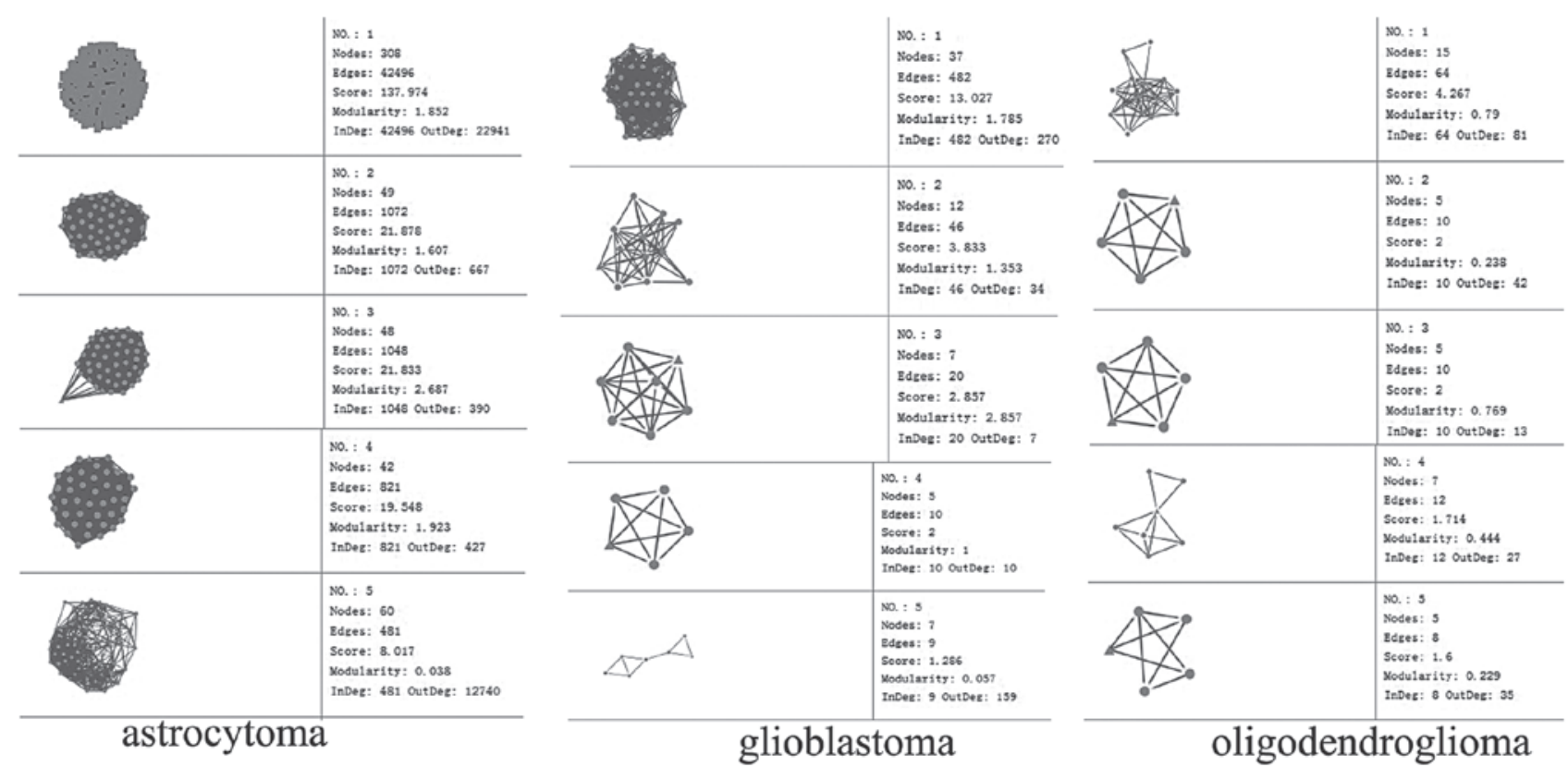

Figure 4. Five modules with the maximum number of genes selected respectively from the networks of the three types of glioma.

previously demonstrated to be implicated in glioma invasion $(28,29)$, and PAK is a potential therapeutic target in schwannomas (30). DOK-6 expression was relatively high in several human neuroblastoma cell lines and detected in the developing neocortex, diencephalon, spinal cord and cranial parasympathetic nerve (31), which suggests that 
it has functional roles in neuronal cells. Understanding signal transmission within neural cells is a crucial issue in neuroscience. Nervous system signaling disorders are known to cause numerous diseases, including Parkinson's disease, Alzheimer's disease and cancer (32). DEGs identified in astrocytoma tissues were mainly associated with synaptic signaling, thus novel drugs may be identified that interfere with these pathways.

CD86, a co-stimulatory molecule, is downregulated in glioblastoma to evade immune recognition (33). WNK2 acts as a recurrent target for epigenetic silencing in glia-derived brain tumors and is associated with tumor cell invasion and proliferation (34). One study indicated that the loss of WNK2 expression may contribute to the highly invasive phenotype of glioblastomas and it is important for tumor cell survival and cell cycle progression (35). Protein phosphorylation is involved in the majority of cellular processes and thousands of distinct phosphorylation events may be detected in a single cell type (36). Enrichment analysis of the genes identified through the kinase-substrate network approach suggested that genes encoding phosphorylated signaling hubs are often disease-associated genes, and genes encoding conserved phosphoproteins are associated with cancer (37). Phorbol esters are tumor promoters and mitogens, whose effects may be mediated by changes in ion transport across membranes. They activate the physiological amiloride- and cAMP-sensitive channels gating apical $\mathrm{Na}^{+}$entry from the mucosal medium into the epithelial cells, which is associated with tumor occurrence $(38,39)$. DEGs in the glioblastoma tissues were mainly associated with protein phosphorylation and sodium ion transportation, thus further studies are required to determine whether the genes identified in this study may be targeted clinically in the treatment of glioma.

It has been demonstrated that Cdk5 (40) and DOK-6 (31) were upregulated in oligodendroglioma tissues and have roles in the occurrence of oligodendroglioma. Recent studies have demonstrated that organic acids are cytotoxic against numerous human tumor types, including neuroectodermal and malignant brain tumors $(41,42)$. With regard to its anticancer properties, betulinic acid (a type of organic acid) was previously reported to exhibit selective cytotoxicity against several melanoma-derived cell lines (41). Due to its selective cytotoxicity against tumor cells and favorable therapeutic index, betulinic acid is a highly promising novel chemotherapeutic agent for the treatment of cancer (43). DEGs in oligodendroglioma tissue were mainly those associated with the synthesis of organic acids, and this analysis may aid in the development of novel treatment strategies for the disease.

Reasonable use of microarray gene expression profiling not only renders the analysis of large quantities of biological information quick and simple, but also facilitates the accurate identification of the key molecular mechanisms associated with glioma biological behavior. In the present study, it was demonstrated that distinct treatments should be administrated for the three types of glioma, due to their different functions analyzed from the mined network modules. Furthermore, the results of the present study implied that PAK3 and DOK-6, CD86 and WNK2, and Cdk5 may possibly be the target genes relevant to astrocytoma, glioblastoma and oligodendroglioma respectively. This may be useful in investigating the complex interacting mechanisms underlying the disease, and provides a novel strategy, which may aid with the development of novel therapies for the different types of glioma. However, further studies are required to confirm these results and determine their clinical utility.

\section{Acknowledgements}

The authors would like to thank Fenghe (Shanghai) Information Technology Co., Ltd. for their ideas and help, which provided a valuable added dimension to the study.

\section{References}

1. Chen $\mathrm{H}$, Chen Y, Zhao Y, et al: Association of sequence variants on chromosomes 20,11, and 5 (20q13.33, 11q23.3, and $5 \mathrm{p} 15.33)$ with glioma susceptibility in a chinese population. Am J Epidemiol 173: 915-922, 2011.

2. Parney IF: Basic concepts in glioma immunology. Adv Exp Med Biol 746: 42-52, 2012

3. Huse JT and Holland EC: Targeting brain cancer: advances in the molecular pathology of malignant glioma and medulloblastoma. Nat Rev Cancer 10: 319-331, 2010.

4. Rodenhiser D. and Mann M: Epigenetics and human disease: translating basic biology into clinical applications. CMAJ 174: 341-348, 2006.

5. Cobbs CS: Evolving evidence implicates cytomegalovirus as a promoter of malignant glioma pathogenesis. Herpesviridae 2: $10,2011$.

6. Van Meir EG, Hadjipanayis CG, Norden AD, et al: Exciting new advances in neuro-oncology: the avenue to a cure for malignant glioma. CA Cancer J Clin 60: 166-193, 2010.

7. Stupp R, Mason WP, van den Bent MJ, et al: Radiotherapy plus concomitant and adjuvant temozolomide for glioblastoma. N Engl J Med 352: 987-996, 2005.

8. Nibbe RK, Koyutürk M, and Chance MR: An integrative -omics approach to identify functional sub-networks in human colorectal cancer. PLoS Comput Biol 6: e1000639, 2010.

9. Zhu XL, Ai ZH, Wang J, Xu YL and Teng YC: Weighted gene co-expression network analysis in identification of endometrial cancer prognosis markers. Asian Pac J Cancer Prev 13: 4607-4611, 2012.

10. García-Claver A, Lorente M, Mur P, et al: Gene expression changes associated with erlotinib response in glioma cell lines. Eur J Cancer 49: 1641-1653, 2013.

11. Chen J, Ma M, Shen N, Xi JJ and Tian W: Integration of cancer gene co-expression network and metabolic network to uncover potential cancer drug targets. J Proteome Res 12: 2354-2364, 2013.

12. Laenen G, Thorrez L, Börnigen D and Moreau Y: Finding the targets of a drug by integration of gene expression data with a protein interaction network. Mol Biosyst 9:1676-1685, 2013.

13. Morine MJ, Toomey S, McGillicuddy FC, et al: Network analysis of adipose tissue gene expression highlights altered metabolic and regulatory transcriptomic activity in high-fat-diet-fed IL-1RI knockout mice. J Nutr Biochem 24: 788-795, 2012.

14. Ho S, Chao Y, Tong W and Yu S: Sugar coordinately and differentially regulates growth-and stress-related gene expression via a complex signal transduction network and multiple control mechanisms. Plant Physiol 125: 877-890, 2001.

15. Barrett T, Suzek TO, Troup DB, et al: NCBI GEO: mining millions of expression profiles - database and tools. Nucleic Acids Res 33: D562-D566, 2005.

16. Sun L, Hui AM, Su Q, et al: Neuronal and glioma-derived stem cell factor induces angiogenesis within the brain. Cancer Cell 9: 287-300, 2006.

17. Smyth GK: Linear models and empirical bayes methods for assessing differential expression in microarray experiments. Stat Appl Genet Mol Biol 3: 3, 2004.

18. Harris MA, Clark J, Ireland A, et al: The Gene Ontology (GO) database and informatics resource. Nucleic Acids Res 32: D258-D261, 2004.

19. Dennis G Jr, Sherman BT, Hosack DA, et al: DAVID: database for annotation, visualization, and integrated discovery. Genome Biol 4: P3, 2003. 
20. Hong S, Dong H, Jin L and Xiong M: Gene co-expression network and functional module analysis of ovarian cancer. Int $\mathrm{J}$ Comput Biol Drug Des 4: 147-164, 2011.

21. Pyon YS, Li X and Li J: Cancer progression analysis based on ordinal relationship of cancer stages and co-expression network modularity. Int J Data Min Bioinform 5: 233-251, 2011.

22. Praneenararat $T$, Takagi $T$ and Iwasaki W: Integration of interactive, multi-scale network navigation approach with cytoscape for functional genomics in the big data era. BMC Genomics 13 (Suppl 7): S24, 2012.

23. Bader GD and Hogue CW: An automated method for finding molecular complexes in large protein interaction networks. BMC Bioinformatics 4: 2, 2003.

24. Marumoto T and Saya H: Molecular biology of glioma. Adv Exp Med Biol 746: 2-11, 2012

25. Dougan M, Li D, Neuberg D, et al: A dual role for the immune response in a mouse model of inflammation-associated lung cancer. J Clin Invest 121: 2436-2446, 2011.

26. Berzofsky JA, Terabe M and Wood LV: Strategies to use immune modulators in therapeutic vaccines against cancer. Semin Oncol 39: 348-357, 2012.

27. Tang Y, He W, Wei Y, et al: Screening key genes and pathways in glioma based on gene set enrichment analysis and meta-analysis. J Mol Neurosci 50: 324-332, 2013.

28. Tenan M, Aurrand-Lions M, Widmer V, et al: Cooperative expression of junctional adhesion molecule- $\mathrm{C}$ and -B supports growth and invasion of glioma. Glia 58: 524-537, 2010.

29. Kissil JL, Blakeley JO, Ferner RE, et al: What's new in neurofibromatosis? Proceedings from the 2009 NF conference: new frontiers. Am J Med Genet A 152: 269-283, 2010.

30. Flaiz C, Chernoff J, Ammoun S, Peterson JR and Hanemann CO: PAK kinase regulates Rac GTPase and is a potential target in human schwannomas. Exp Neurol 218: 137-144, 2009.

31. Kurotsuchi A, Murakumo Y, Jijiwa M, et al: Analysis of DOK-6 function in downstream signaling of RET in human neuroblastoma cells. Cancer Sci 101: 1147-1155, 2010.

32. Liao LD, Chang YJ, Lai HY, et al: A novel light-addressable multi-electrode array chip for neural signal recording based on vcsel diode arrays. J Neurosci and Neuroeng 1: 4-12, 2012.
33. Cheema TA, Wakimoto H, Fecci PE, et al: Multifaceted oncolytic virus therapy for glioblastoma in an immunocompetent cancer stem cell model. Proc Natl Acad Sci USA 110: 12006-12011, 2013.

34. Moniz S, Martinho O, Pinto F, et al: Loss of WNK2 expression by promoter gene methylation occurs in adult gliomas and triggers Rac1-mediated tumour cell invasiveness. Hum Mol Genet 22: 84-95, 2013.

35. Moniz S, Martinho O, Reis RM and Jordan P: Protein kinase WNK 2 regulates cell migration in malignant gliomas. http://hdl. handle.net/10400.18/243. Accessed 2011.

36. Hunter T: Regulation of cell signaling by protein phosphorylation, ubiquitylation and SUMOylation. Hong Kong University of Science and Technology, 2012.

37. Tan CSH, Bodenmiller B, Pasculescu A, et al: Comparative analysis reveals conserved protein phosphorylation networks implicated in multiple diseases. Sci Signal 2: ra39, 2009.

38. Civan MM, Rubenstein D, Mauro T and O'Brien TG: Effects of tumor promoters on sodium ion transport across frog skin. Am J Physiol Cell Physiol 248: C457-C465, 1985.

39. Tamai I, Ohashi R, Nezu J-I, et al: Molecular and functional identification of sodium ion-dependent, high affinity human carnitine transporter OCTN2. J Biol Chem 273: 20378-20382, 1998.

40. Kremer D, Aktas O, Hartung HP and Küry P: The complex world of oligodendroglial differentiation inhibitors. Ann Neurol 69: 602-618, 2011.

41. Fulda S: Betulinic acid for cancer treatment and prevention. Int J Mol Sci 9: 1096-1107, 2008

42. Jaggi M, Ramadoss S, Rajendran P and Siddiqui MJA: Betulinic acid derivatives having antiangiogenic activity, processes for producing such derivatives and their use for treating tumor associated angiogenesis. US Patent 6,403,816. Filed September 20 , 1999; issued June 11, 2002.

43. Cichewicz RH and Kouzi SA: Chemistry, biological activity, and chemotherapeutic potential of betulinic acid for the prevention and treatment of cancer and HIV infection. Med Res Rev 24: 90-114, 2004. 